Pecvnia, 6 (2008), pp. 75-105

\title{
Modernización de la Gestión Pública. Necesidad, incidencias, límites y críticas
}

Recibido: Abril 2008

Aceptado: Junio 2008

\author{
Yolanda Fernández Santos \\ yfers@unileon.es \\ José Miguel Fernández Fernández \\ jose-miguel.fernandez@unileon.es \\ Alicia Rodríguez Pérez \\ arodp@unileon.es \\ Universidad de León \\ Fac. de Ciencias Económicas y Empresariales \\ Campus de Vegazana, $\mathrm{s} / \mathrm{n}$ \\ 24071 León (España)
}

Las nuevas circunstancias culturales, informativas y económicas manifestadas, fundamentalmente, a partir de los años 80 a nivel mundial arrastran a la gestión pública tradicional a una transformación de sí misma, dando lugar a lo que se conoce como la Nueva Gestión Pública (NGP). Numerosas Administraciones Públicas se ven inmersas en esta corriente, reformando sus niveles organizativos y formas de actuación con el objeto de alcanzar una mayor eficiencia y eficacia en su gestión. Pero, en bastantes ocasiones, las características particulares de cada país hacen que la implantación de los postulados de la NGP se realice de forma y ritmos distintos, aunque en
The new cultural, informative and economic circumstances mainly shown at a world level, since the beginning of the $80 \mathrm{~s}$, are leading traditional public management to a self-transformation, giving birth to the so called New Public Management (NPM). Many Public Administrations are involved in this stream reforming their organizational levels and ways of performance reaching a bigger efficiency and effectiveness in their management. Nevertheless, on many occasions, the specific characteristics of each country force the introduction of the NPM principles to be applied in a different way and rate, although in some aspects they may 
algunos puntos presenten cierta convergencia. Esta situación ha puesto de manifiesto la existencia de límites en la aplicabilidad de la NGP y ha originado la crítica seria y razonada de diversos autores en la materia.

Palabras clave: Nueva Gestión Pública, Entidades Públicas, Límites, Críticas. have convergence points. This situation implies the existence of certain constraints as far as the NPM application is concerned and has provoked serious and reasoned objections among the experts.

Key words: New Public Management, Public Organizations, Constraints, Criticism.

\section{INTRODUCCIÓN}

El incremento notable de la presencia e intervención del Estado en los últimos cien años, sobre todo en los países industrializados, se debió, antes de la II Guerra Mundial (1939-1945), a la necesidad de superar los graves daños causados por la Gran Depresión ${ }^{1}$ en los sistemas económicos y sociales, y en la posguerra, a la confianza depositada en la intervención estatal. Su consecuencia más evidente ha sido un cambio de orientación centrado, por una parte, en la ampliación de la capacidad institucional (desde el punto de vista cualitativo no cuantitativo) y, por otra, en la eficacia de la acción estatal para atender las necesidades de la población y el buen funcionamiento de los mercados.

En esta línea, el Banco Mundial considera que el Estado, o de forma más genérica el Sector Público, es necesario para alcanzar el desarrollo sostenible tanto en el plano económico como social, lo que implica que "un Estado eficaz es imprescindible para poder contar con los bienes y servicios $-\mathrm{y}$ las normas e instituciones- que hacen posible que los mercados prosperen y que las personas tengan una vida más saludable y feliz", a lo que hay que añadir la consideración de que "el Estado es fundamental para el proceso de desarrollo económico y social, pero no en cuanto agente directo del crecimiento sino como socio, elemento catalizador e impulsor de ese proceso" (Banco Mundial 1997: 1). En caso contrario, ¿quién solucionaría el fracaso del mercado en relación a la provisión de servicios $\mathrm{y} / \mathrm{o}$ bienes socialmente necesarios; las imperfecciones del mercado; el poder del mercado; las externalidades; la cuestión de la confianza en situaciones donde bienes y consumidores son sensibles y vulnerables, y la equidad y distribución de los recursos?

La denominada "Gran Depresión" fue una crisis económica mundial iniciada en octubre de 1929, con la caída de la Bolsa de Nueva York, que se prolongó durante toda la década de los años 30, siendo particularmente intensa hasta 1934. 
Además, otra de las razones fundamentales que contribuyen a que no se pueda prescindir del Sector Público es que éste o las entidades que lo forman deben aportar a la comunidad económica las bases necesarias para mejorar su competitividad en una economía global. Así, por ejemplo, algunos de los aspectos en los que este Sector es esencial son la mejora de las infraestructuras de comunicación, la preparación básica del capital humano, la agilidad en la toma de decisiones y la eficacia y eficiencia en la prestación de los servicios y en su gestión (Ministerio de Administraciones Públicas-MAP 2000: 34).

A pesar de esta necesidad, existe un considerable consenso ${ }^{2}$ en torno a la idea de que el funcionamiento del Sector Público ha desarrollado una serie de disfunciones que tienen como resultado más significativo la falta de adaptación de las organizaciones públicas al contexto socioeconómico actual, lo que unido al incremento de su tamaño, de la actividad y del gasto público, ha ocasionado un aumento de la demanda de responsabilidad pública por parte de los ciudadanos.

Como consecuencia, la propia Administración Pública se ha planteado la necesidad de trazar una reforma profunda de sí misma, con el objeto de pasar de ser una Administración clasista o asistencialista, basada en la realización de una serie de prestaciones y de servicios gestionados directamente por aquella (supuestamente gratuitos), a una Administración mucho más acorde con el contexto mundial actual (Hufty 1998: 30), esto es, una Administración orientada al ciudadano, el cual, a cambio de sus tributos, exige más información, un número más elevado de productos y servicios, mayor calidad de los mismos y un incremento de la eficiencia en la gestión de los recursos que utiliza, en definitiva, una mejora de su actuación.

En síntesis, significa pasar de la cultura administrativa del gasto a la conciencia del coste, de la cultura del monopolio a la cultura de la competencia, de la cultura del ciudadano-servidor a la cultura del ciudadano-cliente, de la cultura de la burocracia a la cultura de la adaptación, de la flexibilidad, de la preocupación por la productividad y de la calidad en la provisión de los servicios, lo que obliga a la Administración a ser competitiva en el mercado (Vivas Urieta 1998: 35).

\footnotetext{
2 Véase, entre otros, Onrubia Fernández (1995), Sánchez Motos (1997) y Montesinos Julve y Gimeno Ruiz (1998).
} 
De igual forma, Cunill considera que esta transformación de la Gestión Pública ${ }^{3}$ consiste en el reemplazo de:

los métodos burocráticos por métodos manejados por el mercado para la provisión de bienes y servicios producidos por el gobierno. A tal efecto, se trata de constituir mercados para la mayoría de los servicios públicos, separando la elaboración de políticas de la provisión de servicios (o ejecución de políticas). Ello se realiza abriendo éstos a la competencia y desarrollando vínculos contractuales entre los proveedores de los servicios, los consumidores y los financiadores, de manera que las agencias ${ }^{4}$ puedan responder a las presiones del mercado (Cunill 1997: 236).

Esto deriva en un incremento de la preocupación por el cliente en el conjunto del Sector Público y en los distintos niveles de la Administración Pública, con el objetivo de buscar una adecuación de los servicios públicos a las necesidades del ciudadano, con un grado satisfactorio de calidad en la prestación de los mismos, buscando con ello un equilibrio entre el coste y la calidad y asegurando que la Administración no se convierta en rehén del ciudadano al centrarse únicamente en su satisfacción (OCDE 1997: 40).

Este cambio de visión de la Administración Pública, denominada por Hood (1991) como Nueva Gestión Pública (NGP), o en términos anglosajones New Public Management ${ }^{5}$ (NPM), y descrita como una «reinvención del gobierno» por Osborne y Gaebler (1992), ha despertado un interés que supera el ámbito nacional ${ }^{6}$. Tanto es así que han surgido

3 La Gestión Pública podría definirse como "la aplicación de los recursos adecuados para alcanzar los objetivos públicos socialmente deseados; es decir, la obtención de los fines que la sociedad valora con el menor sacrificio de recursos posible" (López Casanovas 1995: 61).

4 El término «agencia» hace referencia a una organización administrativa especializada a la que se confía la gestión de un servicio.

5 En algunas ocasiones la NPM está referenciada en la literatura con otros términos como «gerencialismo» (managerialism) (Pollitt 1990 y Hughes 2003), «Administración Pública basada en el mercado» (market-based Public Administration) (Lan y Rosenbloom 1992: 535), «gobierno emprendedor» (entrepeneurial government) (Osborne y Gaebler 1992), «gerencia corporativa» (corporate management) y «nuevo gerencialismo» (new managerialism) (Boston 1991: 8-9).

6 En un primer momento la NGP tuvo mayor impacto en los países de habla inglesa del mundo desarrollado. El inicio de su desarrollo empírico tuvo lugar en de Reino Unido con la llegada Margaret Thatcher al gobierno inglés en el año 1979, quien promovió el impulso para que los cambios comenzaran a formalizarse, basado todo en la idea de mermar el papel y tamaño del Estado. El siguiente país fue Estados Unidos bajo el mandato de Reagan (1980), después Nueva Zelanda y, por último, gran parte de los países de la OCDE.

Salvador Serna (2001: 84-86) enumera las circunstancias que en su opinión explican el hecho de que la doctrina de la NGP emergiera inicialmente en los países anglosajones. 
distintos organismos públicos internacionales que se han implicado en la materia, como el Fondo Monetario Internacional (FMI), el Banco Mundial (BM) y, en particular, la Organización para la Cooperación y el Desarrollo Económico (OCDE).

En general, estas instituciones han puesto de manifiesto la necesidad de llevar a cabo una transformación intensa de la Administración Pública, mediante la revisión de sus fines y formas de funcionamiento y la creación de nuevas estructuras organizativas y funcionales que permitan cambiar las políticas y costumbres para adecuarse a los cambios rápidos que se están produciendo en la sociedad (Muñoz Colomina 1996: 81).

De esta forma, la NGP es presentada como una reacción práctica frente a la creciente tendencia a la burocratización de las sociedades, a la insatisfacción de los ciudadanos con bienes y productos suministrados por organismos estáticos, al déficit público y a la dificultad que tienen los ejecutivos políticos de llevar a la práctica las reformas que impulsan las organizaciones burocráticas (Hufty 1998. 29).

\section{UNA NECESIDAD MANIFIESTA \\ 2. MODERNIZACIÓN DE LA GESTIÓN PÚBLICA TRADICIONAL:}

La Gestión Pública Tradicional llevada a cabo por los países desarrollados en el siglo XX, apenas presentaba problemas dadas las características del Sector Público y de su entorno; ahora bien, el cambio reciente en dicho entorno ha puesto de manifiesto la necesidad urgente de renovar la organización y las normas administrativas con el objetivo de conseguir una Administración Pública más moderna y competitiva. La base que propicia estos cambios está apoyada principalmente en el ahorro y reducción de costes, en la evolución de las tecnologías y en la mejora de los servicios públicos.

Los tres motivos que enumera Winberg (1994: 85-86) para iniciar las reformas que resultan esenciales en la gestión pública son:

- Falta de recursos en el sistema. Esto implica la imposibilidad de continuar haciendo las cosas tal y como se venían haciendo antes, lo que supone eliminar la realización de actuaciones innecesarias (despilfarros) que conllevan costes para la entidad.

- Dar respuesta a la situación internacional de globalización a través de la mejora en los servicios. Una Administración Pública debe 
aprovechar los recursos disponibles para prestar el mejor servicio posible con el objetivo de prosperar en el entorno competitivo en el que se asienta.

- Rápido ritmo de cambio provocado no sólo por la competitividad internacional sino por las innovaciones en comunicación e informática, que propician una mayor rapidez en la toma de decisiones.

A estas causas hay que añadir otras expuestas por Muñoz Colomina (1996: 81), como son: la competencia existente en el mercado; la necesidad de conocer el coste real de los servicios prestados para tener un punto de referencia a la hora de fijar las tasas; la obligación de eliminar el aislamiento de los entes públicos con respecto a los privados y de buscar la coordinación de sus actividades con el objetivo de conseguir economías de escala.

No obstante, de todos estos motivos, el más importante que hay que destacar en la gestión de los organismos públicos es la reducción de recursos financieros ocasionada por el control del déficit público 0 , como bien indica Aucoin (1997: 493), por la necesidad gubernamental de responder a una situación de crisis fiscal derivada de los cambios en el sistema económico internacional y de la creciente demanda de servicios y de regulaciones que reciben los sistemas políticos nacionales, autonómicos o locales.

Todos los factores expuestos anteriormente, que explican el origen del conjunto de reformas de modernización incluidas bajo la denominación NGP, se podrían agrupar en tres categorías (Fuertes Fuertes 2004: 34-37): factores económicos, factores de carácter ideológico y factores tecnológicos.

- Factores económicos. Hacen referencia a la necesidad de ajustar los presupuestos tras la crisis del petróleo en los años setenta, a los problemas financieros con los que deben enfrentarse los gobiernos y a los desequilibrios presupuestarios.

Así, surge la necesidad política en los distintos organismos de llevar un control de eficacia y eficiencia, a través de distintas actuaciones, como pueden ser el control más riguroso del gasto o la introducción de reformas en el sistema de financiación local al enfrentarse a la necesidad de «hacer más con menos dinero», etc. 
Por otro lado, el proceso de globalización actúa como fuerza de presión para la modernización de la Administración Pública, aumentando el interés de los países por la competitividad económica y la evaluación de sus actuaciones con respecto al resto de los países.

- Factores de carácter ideológico. Aquellos factores que influyen en la modernización de la gestión pública se centran básicamente en: (1) una mayor orientación hacia el ciudadano entendido como cliente, con la posibilidad de elegir libremente al suministrador del servicio y prestando mayor interés a sus necesidades y derechos; (2) una cada vez más clara separación entre la política y la gestión, diferenciando así, tanto sus responsabilidades como el papel a desempeñar por los distintos colectivos (políticos y gestores) y poniendo las tareas ejecutivas en manos de organizaciones creadas expresamente para ello; y, (3) el cambio de la posición ideológica en la división de roles entre el Sector Público y el Sector Privado, a través de nuevas formas de prestación de servicios públicos o fórmulas más cercanas a las técnicas de mercado en competencia con las empresas privadas.

- Factores tecnológicos. Dentro de éstos hay que destacar principalmente el desarrollo de las nuevas tecnologías de la información. La Administración Pública no puede quedar al margen de esta nueva «sociedad de la información», debe modernizarse continuamente adaptándose a las nuevas tecnologías a fin de mantener en todo momento la competitividad, optimizar la calidad de los servicios y mejorar su relación con los usuarios.

De esta forma, el desarrollo de adecuados sistemas de información con las nuevas tecnologías, permitirá intercambiar con fluidez información para una rápida toma de decisiones, una mejor relación entre el ciudadano y la Administración y una mayor coordinación de todos los servicios.

Todos estos factores incentivaron la necesidad de modernización de la Administración Pública, donde el contexto de cultura administrativa angloamericano, más flexible que el de otros países, favorece la implementación de reformas estructurales en el Sector Público, permitiendo el desarrollo de los postulados de la Nueva Gestión Pública (Pollitt 1990; Hood 1991 y Aucoin 1996).

En este sentido, Dawson y Dargie (2002) definen la NGP como un movimiento, un ámbito de estudio por parte de los académicos, y 
un conjunto de prácticas que pueden observarse en las reformas recientes del Sector Público.

La OCDE (1995: 28 y 1997: 37-50) considera que esta nueva forma de gestión de la Administración Pública se caracteriza por las líneas maestras $^{7}$ que se exponen a continuación:

- Desregulación. Este planteamiento busca la disminución de reglas y normas en el Sector Público, intentando a la vez que las que existan permitan un planteamiento estratégico de la gestión a través de la flexibilidad en su aplicación. Se pretende así conseguir una disminución de la tendencia monopolística y una mayor competitividad en algunos servicios.

Por otra parte, también se busca una mayor orientación hacia los resultados en términos de eficiencia, eficacia y calidad del servicio, poniendo énfasis en el establecimiento de objetivos concretos, determinados y cuantificados de forma razonable que permitan hacer un seguimiento de su grado de consecución.

- Descentralización de los poderes de la gestión. La idea de descentralización supone la creación de unidades (entidades, agencias, etc.) más reducidas, con flexibilidad en las normas a aplicar y abandonadas a merced del mercado. Estas nuevas organizaciones desarrollarán funciones, objetivos y responsabilidades bien delimitados, lo que permitirá hacer un seguimiento más efectivo de sus actuaciones.

- Énfasis en las responsabilidades de los gestores y motivación para la mejora. La exigencia de responsabilidades de gestión en sus diferentes niveles requiere, en primer lugar, conocer la situación de partida de la misma, para posteriormente pasar a definir los objetivos de forma clara y adecuada y a diseñar los procesos de gestión necesarios para alcanzarlos, dentro de los límites de los recursos disponibles.

Esto sirve de base no sólo para exigir responsabilidades a los gestores, sino para incorporar incentivos al personal con el fin de llevar a cabo una mejora continua de la gestión pública. No obstante, exige

\footnotetext{
7 Muchos de estos fundamentos han sido desarrollados por Montesinos Julve (1996 y 1998), Pablos Rodríguez (1997), Aucoin (1997), Montesinos Julve y Gimeno Ruiz (1998), Falconer (1999) y Hughes (2003), entre otros.
} 
que los responsables de la prestación de los servicios públicos sean personas proactivas y no reactivas.

- El reforzamiento de las capacidades estratégicas del centro, junto con la reorganización e implantación de la función pública en la gestión y las reformas. Generalmente, las burocracias se consideran torpes e ineficaces, dotadas de capacidad de innovación y adaptación limitada, en las que es difícil distinguir objetivamente lo político de lo administrativo (Hufty 1998: 24). Por el contrario, la NGP facilita desarrollar capacidades estratégicas que permiten tanto la evolución de la Administración Pública como su adaptación de manera automática, flexible y económica a cambios externos y la respuesta a intereses diversos; en definitiva, permite su transformación en un Sector Público menos burocratizado.

Por otra parte, las distintas organizaciones deben adoptar mecanismos para incentivar a sus empleados, tal como ocurre en el Sector Privado. Para ello es necesario buscar un equilibrio entre la independencia y la profesionalidad de los funcionarios, a través de la sustitución de la norma de contratos públicos, estatuto de funcionarios y leyes sobre el patrimonio, por legislación mercantil, laboral y civil, en beneficio de una mayor flexibilidad en materia de contratación, salarios y uso del patrimonio con criterios empresariales, pero siempre teniendo presente el interés general.

- Gestión más orientada hacia el cliente. El cambio demográfico derivado de la evolución económica y social provoca, por un lado, aumento de la demanda de servicios públicos y, por otro, incremento del peso específico del ciudadano-cliente en la toma de decisiones y en las acciones del organismo público.

Los ciudadanos exigen que la gestión llevada a cabo en el Sector Público responda a sus necesidades y preferencias, y que las decisiones relativas a la distribución de los recursos y a la provisión de los servicios se tomen lo más cerca posible de los destinatarios, creando así un proceso de retroalimentación a través del conocimiento de la reacción de los clientes y otros grupos interesados. Este proceso de feedback ayuda, tanto a las entidades a corregir sus disfunciones, como a los ciudadanos a mantener un juicio fundado y maduro sobre la gestión desarrollada.

- La introducción de la competencia y el mercado. La introducción de los mecanismos del mercado dentro de la gestión pública como 
clave para conseguir menores costes y mejores resultados, donde la competencia se convierte en un factor de comparación que sirve para la evaluación privada y pública de las organizaciones y que debe conducir, en un entorno más amplio, a diferenciar aquellas que funcionan correctamente de aquéllas otras que no lo hacen.

- Utilización de métodos de evaluación y técnicas de gestión aplicadas en el ámbito empresarial. Los organismos que forman el Sector Público deberán incrementar la aplicación de rigurosas medidas de gestión a través de mecanismos disciplinares más cercanos al mundo empresarial. Además, contribuirán a que las distintas entidades se centren en sus específicas responsabilidades y a la realización de tareas lo más eficientes y eficaces posibles. Por lo que estas entidades tendrán que comprometerse a seguir un proceso de mejora continua tanto en los niveles organizativos como en la calidad de los servicios prestados.

En el Gráfico 1 se recoge el resumen del estudio comparativo entre las características de la Gestión Pública Tradicional y la Nueva Gestión Pública.

Esta modificación de perspectiva de la gestión pública ha comprometido a todos los países miembros de la OCDE en un proceso difícil de cambio cultural, poniendo énfasis en:
(...) organizar el gobierno en grupos de agencias y departamentos (...); adoptar decisiones estratégicas y orientadas a la obtención de resultado; utilizar objetivos de output, indicadores de rendimiento o gestión, pagos en relación con los resultados y medidas de mejora de la calidad; recortar los gastos (...); una mayor flexibilidad; una mejora de la eficiencia en la prestación de servicios públicos; la promoción de la competencia en el estado y entre organizaciones del Sector Público (Suleiman 2000: 4).

En definitiva, se trata de alcanzar una estructura institucional flexible que favorezca la transparencia y la rendición de cuentas, que se centre en la mejora de los resultados de las intervenciones públicas en vez de en procesos y normas rígidas para la provisión de servicios. Esto contribuye a reformar la gestión pública, propiciando la exploración de nuevas alternativas en la prestación de servicios públicos o la supresión de aquellos innecesarios, así como una orientación hacia el mercado que, sin duda, promueve la competencia y la innovación. 
Gráfico 1: Gestión Pública Tradicional versus Nueva Gestión Pública

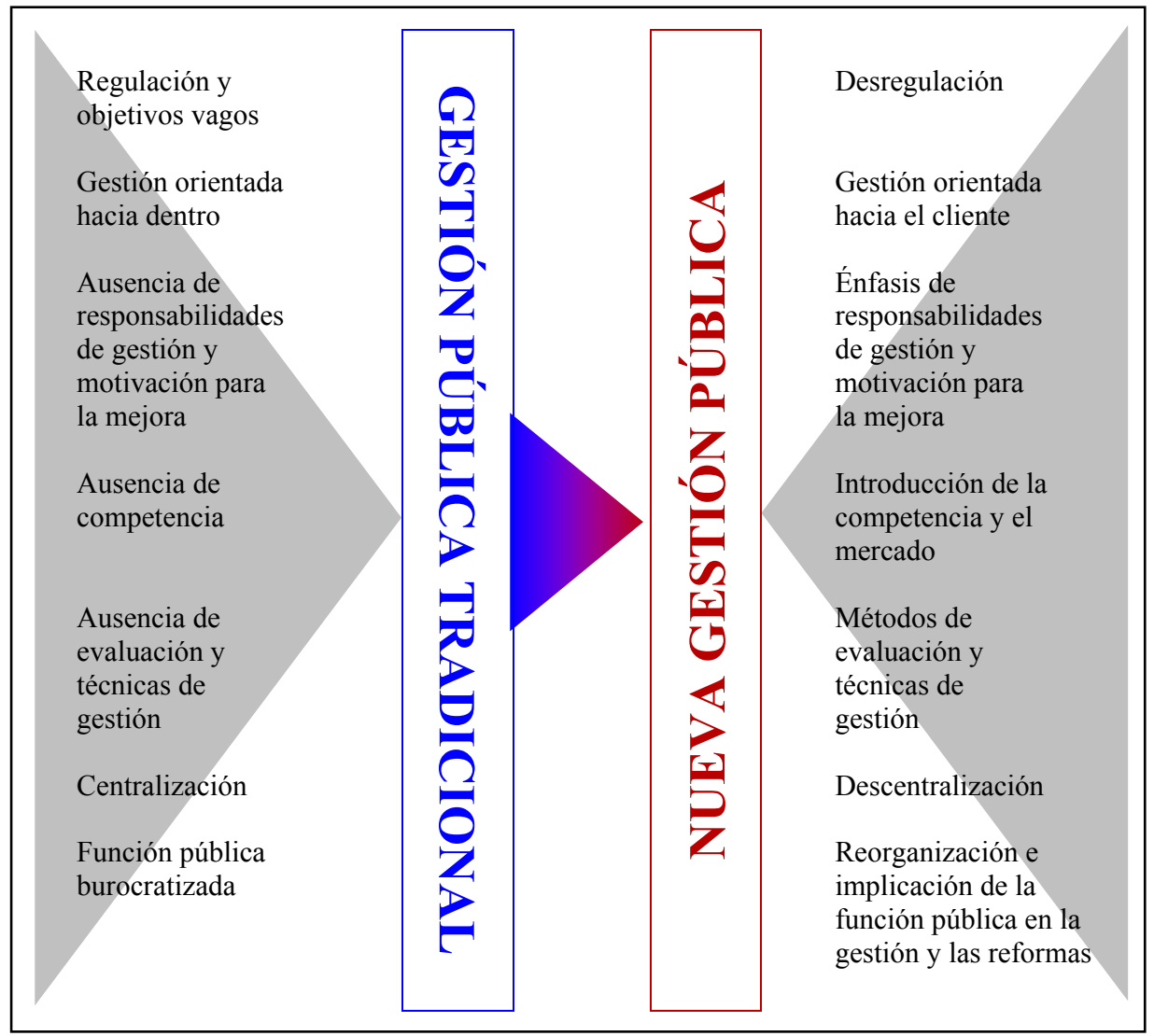

Fuente: Adaptado de Montesinos Julve (1998).

En definitiva, y siguiendo a Hughes (1997: 105 y 2003: 54), la NGP implica pasar del concepto de administrar (seguir instrucciones) al de gestionar (conseguir resultados). Por ello, es necesaria la utilización de técnicas de control que vigilen la actuación de la Administración Pública y verifiquen la consecución de sus fines, donde el personal directo de la misma tenga un papel más activo en el logro de los objetivos preestablecidos.

PÚBLICA

\section{INCIDENCIAS DE LA IMPLANTACIÓN DE LA NUEVA GESTIÓN}

El origen de la NGP (asociada al concepto de «modernización») se remonta a finales de la década de los ochenta como 
consecuencia de los cambios en la Administración Pública, pero básicamente, su aparición en la literatura académica y su desarrollo profundo tuvo lugar en la década de los noventa (Hood 1991; Hood y Jackson 1991; Osborne y Gaebler 1992 y Aucoin 1997). En esta época, se configura una serie de estrategias y herramientas reformadoras, presumiblemente inequívocas, a veces consideradas ambiguas además de irreversibles, convergentes y beneficiosas, que inducen a generalizar una influencia global y un modelo doctrinal dominante en la gestión pública contemporánea (Dollery y Won Lee 2003: 3).

La tendencia hacia la NGP ha suscitado controversias que han dado lugar a numerosos problemas a la hora de su adopción generalizada. Algunos autores ven la NGP como una nueva forma de entender y de llevar a cabo las funciones en el Sector Público, es decir un cambio de paradigma, otros la conciben como una adopción simplista de las peores características de la gestión privada, no teniendo en cuenta las diferencias existentes entre ambos sectores.

Así, por un lado, existen autores como Lynn (1996 y 1998), Boyne (1996) y Kettl (1997) ${ }^{8}$ que ven la «excitación transitoria» sobre la NGP como algo a explorar, esto es, consideran la NGP un tema perecedero con alta probabilidad de desaparecer. Además, ven el estudio de la NGP como una oportunidad para crear mejores modelos teóricos.

Esta última postura es la defendida por Villoria al afirmar que "los discursos modernizadores son inherentemente políticos e ideológicos, y su construcción es el fruto de la conquista de una verdad desde la primacía comunicativa y de poder, no de la conquista científica" (Villoria 1997: 80). Esto significa que el gobierno y la NGP permiten a los estudiosos explorar los determinantes de la política y sus impactos, sin llegar a enredarse en la dicotomía entre explicaciones exageradas y superficiales de los resultados o desempeños (Ewalt 2001: 13).

Por otro lado, están aquellos autores, como Hughes (1997: 104-105), Mathiasen (1999) y organizaciones como la OCDE (1998), que consideran la NGP como un nuevo paradigma de gestión con una larga

Otros autores que no consideran la NGP como un paradigma de cambio son: Pollitt (1990), Hood (1995), Pollitt y Bouckaert (2000) y Gruening (2001). 
vida'. Todos ellos defienden que la NGP ha suplantado al modelo tradicional de gestión pública al que difícilmente se volverá, convirtiendo al Sector Público en gerencial más que administrativo, tanto en la práctica como en la teoría. Esto origina el desarrollo de mecanismos de gestión pública específicos, no quedándose en la simple adopción de prácticas del Sector Privado debido, principalmente, a las diferencias entre ambos sectores.

A pesar de la existencia de diferentes formas de pensar, implementar la NGP no es una tarea fácil, sino que conlleva numerosas dificultades: ¿Cómo implantar la NGP en un determinado país, jurisdicción, Entidad Local, etc., en general entidad pública, para dar solución a los problemas que se plantean?

Para dar respuesta a esta cuestión tenemos que mencionar las diferentes corrientes o formas de gestión que origina la NGP en su afán de modernizar la Administración Pública. Estas corrientes o formas de gestión son, según Ramió Matas (2001): el enfoque neopúblico y el enfoque neoempresarial.

\section{El enfoque neopúblico}

Pone especial atención en la repolitización, la racionalización y el control de la externalización de los servicios públicos, la participación ciudadana y la ética en la gestión pública.

Las características principales de esta corriente son: a) reforzar el concepto de ciudadanía, buscando recrear la legitimidad política; b) potenciar los valores de lo público, creando una cultura en la eficacia y eficiencia y en la ética; c) reconocer nuevos derechos a los ciudadanos; d) conseguir la satisfacción de los ciudadanos; e) actuar con principios como la universalidad y la igualdad y no como el economicismo y la gestión; f) incrementar la calidad y el número de servicios públicos; f) definir claramente aquello que se puede o no se puede externalizar, desde la perspectiva de las necesidades y derechos de los ciudadanos.

No obstante, este enfoque no está exento de críticas y problemas, como son, entre otros, los siguientes:

Otros autores que defienden la idea de que la NGP es un nuevo paradigma de cambio son: Barzelay (1992), Osborne y Gaebler (1992), Holmes y Shand (1995) y Aucoin (1996 y 1997). 
1. los principios básicos de igualdad, participación, cultura pública, regulación y rígido control de los servicios públicos externalizados se utilizan para justificar la imposibilidad de alcanzar una gestión flexible, eficaz y eficiente,

2. la defensa de los valores públicos puede conducir al mantenimiento del modelo burocrático y a la satisfacción de las necesidades de los empleados más que las de la ciudadanía.

\section{El enfoque neoempresarial}

Pone énfasis en la economía, la eficacia y la eficiencia de las entidades públicas, al mismo tiempo que propone una clientelización de los ciudadanos.

Las características más destacadas de la corriente neoempresarial son: a) el Estado Relacional y la sociedad del bienestar, más que el Estado intervencionista y del bienestar; b) fomento del cambio de cultura en el interior para pasar de proveedor a intermediario; c) adopción del lenguaje y los conceptos del Sector Privado; d) acortamiento de las distancias en las relaciones entre Administración y ciudadanía; e) fragmentación en unidades más pequeñas con creciente autonomía en la gestión económica, patrimonial, laboral, etc.; f) materializaron a través de organismos con formas jurídicas alejadas, en mayor o menor grado, del Derecho Público.

También son numerosas las críticas y problemas que suscita este enfoque de la NGP, entre los que pueden destacarse:

1. confusión de medios con fines, al considerarse por sí mismos tanto indicadores como actuaciones modernizadoras;

2. formulación de objetivos sin compromisos concretos, de difícil verificación por la ciudadanía;

3. consideración de que la gestión privada es la solución de los problemas;

4. reducción de la condición de los ciudadanos a simples clientes;

5. atenuación de las responsabilidades como consecuencia de su origen de organismos alejados, en mayor o menor medida, del Derecho Público;

6. las obligaciones se mantienen o aumentan, mientras los derechos disminuyen; 
7. incremento de los baremos para la utilización de los servicios públicos dando lugar, en ocasiones, a la exclusión de determinados colectivos de ciudadanos, en aras de obtener un buen indicador económico relacionado con la reducción de costes.

La corriente o forma de gestión pública ideal sería un híbrido entre los dos enfoques, reduciéndose así, los problemas de ambos modelos. No obstante, si hay que decantarse por uno de ellos se haría por el enfoque neoempresarial, a pesar de las críticas y problemas que ello conlleva. La razón de esta elección es que muchos de los problemas enumerados en el mismo pueden evitarse o suavizarse controlando los niveles más bajos de la Administración Pública por aquellos responsables de su aplicación, que en gran parte son los gestores de los servicios públicos. Sin embargo, en el enfoque neopúblico, los problemas son más difíciles de resolver, ya que no dependen tanto de los gestores responsables de los servicios públicos como de niveles más elevados en el ranking organizativo.

Así, una vez analizada la situación de la entidad pública donde se quiere actuar para mejorar la gestión y elegido uno de los dos enfoques expuestos anteriormente, el problema que se plantea es cómo llevar a la práctica el establecimiento de la NGP.

En este sentido, Ewalt afirma que "la aplicabilidad y efectividad de los conceptos de la NGP variarán considerablemente de un país a otro y de una jurisdicción a otra" (2001: 11 y 13).

Por lo que respecta a las diferencias que puedan surgir del establecimiento de la NGP en los distintos países, Flynn (2002) argumenta que éstas pueden provenir del grado de transferencia de la gestión de los servicios públicos al Sector Privado, del modo de llevar a cabo la descentralización, de la evolución del control del input al control del output que pueda existir, de la manera de realizar la selección y promoción del personal, de la forma de llevar a cabo la política de orientación al cliente, etc.

Además, esta aplicabilidad también depende, entre otras causas, de la etapa concreta de desarrollo en la que se encuentre el país, de su nivel de ingresos, su tamaño, configuración étnica, características culturales y sistemas políticos y administrativos. Esto supone la consideración de cada entidad como un ente único. En consecuencia, la NGP no es un simple modelo de gestión pública, sus reformas deben hacerse teniendo 
en cuenta las diferencias nacionales y las circunstancias locales, pudiendo implicar el establecimiento de técnicas operativas diferentes, reformas profundas o selectivas, en cuyo proceso se podrán aplicar distintas estrategias.

En definitiva, esta nueva situación presenta un cambio frente a anteriores reformas. La teoría y los detalles del programa están más claros que en otras ocasiones, en las que se perseguía reducir costes. Esta reforma no consiste en una especialización técnica dentro de la Administración Pública, sino en reemplazar el modelo tradicional en su conjunto, en otras palabras, la NGP representa una transformación del Sector Público y sus relaciones con el gobierno y la sociedad (Hughes 2003: 50).

La aplicación de la NGP está lejos de haber acabado y su proceso de implantación ha desencadenado en una serie de problemas en los distintos países, administraciones o entidades, entre los que se destacan:

- La desigualdad del ritmo y extensión de las transferencias de competencias.

- La posible desaparición de la visión de conjunto de la función pública del Estado y de los valores tradicionales del servicio público, como consecuencia de la flexibilidad en la gestión derivada de la diversidad de prácticas.

- La dificultad de vincular la recompensa individual al resultado global de la gestión, al no existir una conexión directa entre ambos.

- La problemática de coordinar la misión de la entidad pública con los objetivos operativos de la misma o, incluso, con la medición de los resultados.

- El conflicto en la determinación de los responsables de los resultados (los representantes elegidos o los empleados públicos), en un entorno de gestión descentralizado debido a la creciente autonomía de los mismos.

A pesar de los problemas descritos previamente, este fenómeno denominado NGP, aunque insertado dentro del proceso global de transformación del Estado, se ha expandido a nivel mundial, constituyendo el eje central para el cambio y modernización de las Administraciones 
Públicas. En este sentido, son numerosos los países ${ }^{10}$ con diferentes velocidades de desarrollo y con distintos objetivos y prioridades que se sitúan dentro de la estructura genérica de los postulados de la NGP.

Este proceso de cambio que se está produciendo a nivel internacional y las reformas que se están llevando a cabo inciden con diferente intensidad en los distintos países de nuestro entorno, bien modificando en algunos casos la cultura y manera de proceder (Poole et al. 1995), bien cambiando los principios de gestión en todos o algunos de los ámbitos del Sector Público (Hood 1991; Thomson 1992 y Likierman 1994).

Dollery y Won Lee (2003: 3) manifiestan que, aún existiendo estas incidencias en la realidad y las diferentes opiniones argumentadas, se está tendiendo a una convergencia ${ }^{11}$ de la gestión pública a nivel mundial, lo que no sólo lleva a una aproximación de los sistemas administrativos públicos de los diferentes países, sino que además, implica un acercamiento entre las estructuras organizacionales públicas y sus homólogas privadas.

Sin embargo, la conclusión a la que llegan Torres y Pina (2003) para países de la Europa Continental, es que no existe una tendencia global hacia el modelo de NGP de los países pioneros, debido a que las diferencias entre los estilos de Administración Pública todavía perduran, si bien, reconocen similitudes en aspectos clave como la descentralización, desconcentración e iniciativas en la gestión de la calidad, gestión de los recursos humanos y, en menor grado, en la Contabilidad Pública y en la gestión de los resultados.

No obstante, y a pesar de la ausencia de esta convergencia global en la gestión pública entre los diferentes países, se observa una cierta voluntad por parte de los mismos a igualar los procesos a través de (Dimaggio y Powell 1991):

10 Dentro de los países pioneros e institucionalmente dispares que han puesto en marcha la NGP dentro de Europa están, por ejemplo, los países germánicos (Austria, Alemania y Suiza), los nórdicos (Dinamarca, Finlandia, Noruega y Suecia) y los del sur de Europa (Francia, Italia, Portugal y España). En todos los casos, los sistemas de gobierno de cada país operan en entornos significativamente diferentes. Incluso existen países muy diversos dentro de la OCDE que han implantado la NGP en su Administración como México, Japón, Australia, Estados Unidos o los antiguos países comunistas (Hungría y la República Checa). También hay que añadir, aunque no pertenezcan a la OCDE, algunos de los países de América Latina.

11 El término «convergencia», al que en lo sucesivo se hace referencia, se recoge al principio de este epígrafe, como uno de los puntos ambiguos, que Hood (2000), identifica con la idea de «modernización» de la gestión pública. 
1. la existencia de organizaciones que, por medio de poderes legales o de otro tipo, pueden coaccionar a otras haciéndolas asumir sus propias características y estructuras;

2. la imitación que realizan algunas organizaciones de determinados procesos de otras más exitosas, y que toman como ejemplo la «mejor práctica» («best practice»);

3. una cultura profesional compartida por los dirigentes, que a través de organizaciones diferentes, induce a presiones normativas que se ajustan a un modo común de comportamiento organizacional.

Dentro de los distintos países miembros de la OCDE existe la costumbre de difundir las innovaciones en la NGP a través de la «mejor práctica» de los distintos casos de estudio. De hecho, hay tres fuentes a través de las que se proporciona este conocimiento:

a) La divulgación, por parte de los profesionales, de sus propias propuestas de reforma de la gestión pública o de trabajos anecdóticos sobre estudios empíricos exitosos, que se han calificado de «mejor práctica».

b) El creciente número de premios a la calidad y a la innovación en el Sector Público dentro de los países miembros de la OCDE, que ha permitido identificar casos de organizaciones que prestan servicios públicos bien gestionados.

c) Las ventajas de ciertas organizaciones, como la OCDE, para disponer de información acerca de prácticas innovadoras desarrolladas en los distintos países miembros. Así por ejemplo, el Programme on Public Management and Governance (PUMA) desarrollado por la OCDE, ha contribuido a que estos países puedan estar a la vanguardia en la aplicación de la NGP.

Sin embargo, se plantea la duda sobre si la «mejor práctica» es la solución a iguales o similares problemas surgidos en los distintos países. Desde el punto de vista científico, Löffler (1999: 2) propone la siguiente cuestión: ¿las soluciones conocidas son realmente las mejores o pueden existir otras propuestas que den mejores resultados pero que aún no se conocen?

No cabe duda de que la «mejor práctica» puede no ser la mejor solución y que pueden existir otras soluciones desconocidas que 
generen mejores resultados. Por otro lado, hay que tener en cuenta que la «mejor práctica» no tiene por qué hacer referencia al funcionamiento de toda la organización, sino que puede referirse a diversos aspectos de la gestión de un ente público, como la productividad, eficacia, eficiencia, satisfacción de los clientes, así como la dimensión organizacional, la gestión de recursos humanos, el uso de tecnología de la información, la orientación a los ciudadanos, etc. (Löffler 1999: 3).

En conclusión, cuando un país, organización o entidad pública decida modernizar alguno de los aspectos de su gestión puede realizarlo mediante una de las dos vías siguientes:

1. Buscar aquel ente que haya mejorado su gestión en ese aspecto y que se considere a nivel internacional como la «mejor práctica»;

2. Ser innovador y definir una forma de gestión novedosa que pueda ser considerada por parte del correspondiente organismo como mejor de las existentes hasta el momento, aunque siempre conlleve algo de imitación ${ }^{12}$.

En relación con las Entidades Públicas Locales, Stewart (1986: 51) califica su gestión como activa y con necesidad de transformación, que puede darse tanto en la provisión de servicios, como en la manera en que son suministrados, en la organización o en el trabajo, etc. Por tanto, podemos afirmar que la selección de los puntos de la gestión local que deben modificarse es una labor del gobierno local, que es quien debe tener en cuenta las circunstancias locales existentes en cada momento.

El Sector Público español ha introducido numerosas mejoras asociadas a la NGP dentro de sus instituciones, ya sea en la organización, en su relación con los ciudadanos, en la eficiencia de su gestión, etc., aunque todavía queda un largo camino por recorrer en muchos aspectos. Normalmente, estas reformas dan prioridad a una serie de transformaciones en el interior de la Administración, apoyándose en ideas básicas de gestión del Sector Privado, que son las que promueven la mejora de la gestión del Sector Público.

12 Véase Hood (2000). 


\section{LIMITACIONES Y CRÍTICAS DE LA NUEVA GESTIÓN PÚBLICA}

La NGP como modelo de gestión innovadora no deja de tener críticas. Diferentes autores y organismos han enumerado los efectos positivos o negativos que genera, así como manifestado su acuerdo o desacuerdo con cada uno de ellos.

Este apartado se centra en los aspectos negativos de la NGP, recogiendo las limitaciones que presenta la implantación de la NGP y algunas críticas vertidas sobre esta forma novedosa de gestión.

Todos los principios que caracterizan la NGP podrían ser correctamente implantados en la Administración Pública si no se tuvieran en cuenta las limitaciones de carácter teórico y práctico de la NGP recogidas en el trabajo desarrollado por Merrien (1998: 142-144):

\section{A) Los límites teóricos de la Nueva Gestión Pública}

En primer lugar, los teóricos defensores de la "Teoría de la Elección Pública"13 (Public Choice Theory) pretenden reducir la NGP a una simple tecnología de reforma administrativa, en la cual los funcionarios no buscan el interés general, sino maximizar su utilidad personal, es decir, satisfacer sus intereses personales.

Por otro lado, están aquellos autores comprendidos entre los economistas liberales o neoliberales que consideran que la NGP está asentada sobre dos hipótesis básicas con el objeto de reducir el Estado a la mínima expresión, dando lugar a dos limitaciones: la necesidad de introducir el mercado y la competencia en las Administraciones Públicas y la voluntad de dar poder al usuario de los servicios.

a) Introducción del mercado y la competencia.

Esto presupone que el mercado es la mejor solución, siempre que no se den las siguientes circunstancias: (1) existencia de un número insuficiente de partes contratantes, (2) condiciones de producción del servicio demasiado complejas e inciertas, (3) estructura rígida de las inversiones, (4) existencia de numerosas eventualidades que condicionan

13 La "Teoría de la Elección Pública" se encarga de la agregación de las preferencias individuales en una regla de elección colectiva. El padre de la "Teoría de la Elección Pública" es James M. Buchanan Jr., Premio Novel de Economía en 1986. 
el trabajo, y (5) oportunismo de los agentes con resultado de un aumento considerable de sus costes de actuación (Williamson 1985: 143).

Las condiciones anteriores son difíciles de reunir en el Sector Público, por lo que se cuestiona si es válida la introducción del mercado como principio para llevar a cabo esta reforma.

b) Dar poder a los usuarios de los servicios públicos.

Aunque la participación de los usuarios está implícita en esta nueva filosofía (NGP), en realidad es imposible que éstos puedan influir realmente en las decisiones de la organización.

\section{B) Los límites prácticos de la Nueva Gestión Pública}

Dentro de los límites prácticos destacan:

a) La descentralización de funciones y la delegación de poder a los agentes autónomos.

La descentralización de funciones genera críticas, pues tiende a sobrecargar las organizaciones operativas con múltiples $\mathrm{y}$, a veces, inconsistentes objetivos, creando una estructura desorganizada que a menudo avanza en diferentes direcciones al mismo tiempo (Aucoin 1997: 503).

Por su parte, la delegación de poder en los agentes autónomos lleva a que las autoridades no puedan desarrollar actividades de coordinación y planificación de la acción pública. En consecuencia, aquéllos se vuelven cada vez más independientes, generan su propia cultura organizacional y se manifiestan reticentes a asumir las directrices centrales.

b) La reforma de la gestión pública debilita el principio de «rendición de cuentas» al no existir, tal como se indica en el punto precedente, una coordinación entre la acción pública y los sistemas de información utilizados en cada nivel o agente autónomo.

La idea de instaurar la NGP en la Administración Pública, de volverla más transparente, más abierta a los usuarios, más innovadora y menos centralizada genera consecuencias negativas derivadas de su actuación. Algunos informes de la OCDE destacan: 
- La mayor flexibilidad en la gestión ha potenciado la diversidad de prácticas y el temor de ver progresivamente desaparecer toda visión de conjunto de la función pública, el sentido de Estado y los valores tradicionales de servicio público.

- La autonomía creciente de los gestores en un entorno descentralizado incide sobre la responsabilidad pública, pues exige determinar quién es el responsable y de qué resultados.

De la misma forma, Gimeno Ruiz (1997: 55) comenta que, a pesar de las bondades aparentes de la NGP, ésta plantea una serie de cuestiones que preocupan a quienes la critican, entre las que destacan:

- El excesivo énfasis en los criterios técnico y racional, dejando en un segundo plano los de carácter político e intuitivo.

- El aumento de la autonomía de gestión supone un desplazamiento del control político y con ello el paso de la responsabilidad pública a una responsabilidad interna.

- El abuso de indicadores de performance cuantitativos, puede desplazar principios básicos del Sector Público, como el servicio a los ciudadanos o la equidad en la prestación de servicios públicos.

En cuanto a las críticas de la NGP hay que destacar en primer lugar la realizada por Pollitt (1993), quien considera que la NGP defiende excesivamente la utilización de las prácticas del Sector Privado en el ámbito público, como si éstas fueran la solución a todos los problemas de la gestión pública.

Por su parte, Hughes (2003: 62-65), a pesar de estar convencido de la mejora que supone la instauración de la NGP, admite la validez de algunas críticas:

1. La base económica de la NGP. La aplicación de la Teoría Económica al Sector Público está mal concebida, puesto que no todos los individuos se comportan racionalmente y no todos los problemas gubernamentales son susceptibles de resolución a través de técnicas económicas, ya que éstas pueden presentar limitaciones ante ciertos problemas públicos.

2. La base en la gestión privada, debido a las grandes diferencias que existen entre el Sector Privado y el Sector Público, como por ejemplo la dificultad de determinar objetivos y medir resultados en el Sector 
Público, la ausencia de un indicador de beneficio para medir la gestión realizada en el mismo, etc. Si bien, es cierto que muchos de los principios organizativos utilizados en la Administración Pública provienen del Sector Privado.

3. El "Neo-Taylorismo", reprochando la vuelta a la gestión científica basada en la estandarización del trabajo, dejando de lado las relaciones humanas.

4. La politización. Una vez reconocida la importancia de la política en la Administración, la politización no debe ser un gran problema, no obstante, un exceso de la misma acarrearía una excesiva politización de las actuaciones públicas.

5. La escasa responsabilidad. Todavía no están claros los límites y tareas de los miembros de la organización, a pesar de que existe una mayor transparencia en la gestión pública y se ha incrementado la responsabilidad respecto al sistema anterior.

6. Dificultades con el contracting-out. La implantación del contractingout no es una tarea sencilla para el Sector Público, pero su consideración por parte de los gestores públicos como herramienta útil para la gestión de servicios públicos puede generar disminución de costes y beneficios para la entidad.

7. Necesidad de normas éticas. La NGP asume una cultura de honradez. Tal vez, al ofrecer mayor transparencia puedan detectarse más fácilmente comportamientos no éticos o corruptos que en el pasado.

8. Implantación y problemas morales, ya que el trabajo de establecimiento de la NGP es diferente al de pensar que es buena idea. En este sentido, la cúpula de las entidades ha realizado esfuerzos, pero sin prestar la suficiente atención a la implantación, falta de adiestramiento del personal y medios, y existe una mala percepción de los cambios por el ambiente de incertidumbre en que se han producido.

9. Especificación confusa de lo que significa la NGP o «managerialism». En algunos países dichos términos llegan a ser en algunos aspectos despectivos, particularmente en Gran Bretaña, donde existe cierta hostilidad hacia éstos a favor del término «managerialist reform», que traducido al español significa «reforma de la gestión». 
Para finalizar, Dunleavy y Hood (1994: 11-13) estructuran las críticas desde un punto de vista cultural, considerando cuatro modalidades: fatalista, jerárquica, individualista e igualitaria (Tabla 1).

Tabla 1: Críticas a la Nueva Gestión Pública

\begin{tabular}{|l|}
\hline \multicolumn{1}{|c|}{ CRÍTICA FATALISTA } \\
\hline La NGP no puede hacer frente a los dilemas \\
básicos de la Administración Pública. \\
Alternativa: \\
Ninguna. \\
Sobrevalorar los sistemas de gestión los hace \\
vulnerables a los ataques fatalistas. \\
\hline \multicolumn{1}{|c|}{ CRÍTICA INDIVIDUALISTA } \\
\hline $\begin{array}{l}\text { La NGP corre el riesgo de convertirse en un } \\
\text { sustitutivo mediocre de los derechos contractuales } \\
\text { individuales. } \\
\text { Alternativa: } \\
\text { Más contratos reales y menos cuasicontratos, } \\
\text { más privatización y menos corporatización. }\end{array}$ \\
\end{tabular}

Fuente: Dunleavy y Hood (1994: 11).

\section{CRÍTICA JERÁRQUICA}

La NGP puede erosionar la cohesión del sistema y situar el Sector Público fuera de control.

Alternativa:

Fortalecer la capacidad central e incrementar las obligaciones de consulta.

\section{CRÍTICA IGUALITARIA}

La NGP puede fomentar la corrupción, acomodarse a los intereses personales de los altos funcionarios y debilitar las responsabilidades.

\section{Alternativa:}

Más cesión de poder a los ciudadanos, más mecanismos anticorrupción, extensión del modelo del rol empresarial, imposición de un control estricto sobre los posibles fraudes en los contratos.

No obstante, a pesar de las limitaciones o críticas que muestra la NGP y reconociendo que este modelo no es homogéneo ni único, es necesario plantearse cuáles son las posibles alternativas de la gestión pública a tener en cuenta en el futuro (Tabla 2).

Así, el modelo de "Estado burocrático" se asocia a un Sector Público específico, con unas competencias básicas y con unas formas de operar distintivas y bien definidas. Por otra parte, el "Estado mínimocomprador" representa un modelo donde las tareas del gobierno consisten en contratar y donde las grandes empresas privadas dominan la provisión de los servicios públicos. Después está el "Modelo descabezado" donde los servicios son a la vez gestionados en defecto y en exceso, es decir, excesivamente gestionados en el nivel de las organizaciones individuales, pero conjuntamente sub-gestinados, ya que no existe un sistema de dirección. Por último, está el "Modelo de reja cerrada" donde no se da una clara diferencia entre los proveedores privados y los públicos; incluso muchos de los servicios públicos pueden prestarlos corporaciones privadas. Además, al mismo tiempo, existen normas de procedimiento fuertes y 
completas, quedando delimitada la provisión efectiva de servicios por las reglas.

Tabla 2. Alternativas para la gestión pública

\begin{tabular}{|c|c|c|c|}
\hline & \multicolumn{2}{|c|}{ Grado de separación entre el Sector Público y el Privado } \\
\hline & & Nivel bajo & Nivel alto \\
\hline \multirow{2}{*}{ 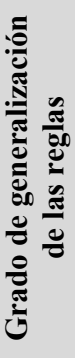 } & 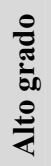 & $\begin{array}{l}\text { Modelo de reja cerrada } \\
\text { Proveedores privados, manuales de } \\
\text { procedimientos (juridificación), sin } \\
\text { mediación política. }\end{array}$ & $\begin{array}{l}\text { Estado burocrático } \\
\text { Extender la provisión pública a través de } \\
\text { organizaciones públicas específicas. }\end{array}$ \\
\hline & 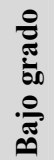 & $\begin{array}{l}\text { Estado mínimo-comprador } \\
\text { Máxima presencia corporativa, el Estado } \\
\text { como "consumidor inteligente". }\end{array}$ & $\begin{array}{l}\text { Modelo descabezado } \\
\text { Sector Público específico pero turbulento, } \\
\text { sin gestores responsables. }\end{array}$ \\
\hline
\end{tabular}

Fuente: Dunleavy y Hood (1994: 14).

Pero, a pesar de todo, la NGP se centra en la voluntad de imponer los valores y modos de funcionamiento de la empresa privada en todas las partes de la Administración Pública, al igual que en la prestación de servicios, de forma abierta a las demandas de los clientes/ciudadanos.

\section{CONCLUSIÓN}

La necesidad de intervención por parte del Sector Público en la economía de cada país se justifica por el conocimiento de fallos en el mercado, como la reducida competencia en algunos sectores, las externalidades que generan determinadas actuaciones, los mercados incompletos, el paro, la inflación, los desequilibrios sociales y ambientales, la distribución desigual de la renta y riqueza, entre otros. El objetivo de dicha mediación es disminuir los efectos negativos que tales situaciones producen en la economía y, como consecuencia, en el bienestar de los ciudadanos.

La Nueva Gestión Pública es una respuesta a la necesidad de adaptación de la Administración Pública al entorno socioeconómico reciente, puesto que el Modelo Tradicional de gestión pública no responde a los cambios que se van produciendo en los distintos países. Esta nueva forma de gestión origina un cambio de orientación y de cultura dentro de 
la organización, dirigida hacia el ciudadano como cliente y está basada principalmente en el ahorro y reducción de costes, en la evolución de las tecnologías y en la mejora de la cantidad y calidad de los servicios públicos.

La introducción de la Nueva Gestión Pública en las Entidades Públicas Locales, como entidades prestadoras de servicios y más próximas a los ciudadanos, supone una repercusión en la concepción propia de la entidad, tanto en la organización como en la forma de actuación, contribuyendo a que sean más claras, más abiertas a los usuarios, más innovadoras y menos centralizadas.

No obstante, la aplicabilidad y efectividad de los postulados que configuran la NGP es una tarea ardua para las Administraciones Públicas de los distintos países, incidiendo con diferente intensidad en los mismos, incluso, pudiendo variar considerablemente de un país a otro y de una jurisdicción a otra. Esto significa que los fundamentos de la NGP no deben tratarse como un modelo universal, puesto que cada país tiene un entorno político e ideológico determinado.

Estas diferencias y dificultades que surgen en la instauración de la NGP se deben fundamentalmente a las características culturales, económicas y administrativas de cada entidad pública, dando lugar a convergencias y divergencias en diversos aspectos de su gestión.

Todo esto ha originado que numerosos autores hayan plasmado sus opiniones, considerando a la NGP como algo efímero con tendencia a desaparecer (Lynn 1998; Boyne 1996; Pollitt 1990, entre otros) frente a otros que la entienden como un nuevo paradigma de cambio con una larga vida (Hughes 1997; Barzelay 1992; Aucoin 1997, entre otros).

También, esta forma de gestión pública innovadora ha suscitado numerosos problemas y críticas al estimar que la implantación de los fundamentos de la NGP tales como la adopción de métodos de gestión del Sector Privado, la introducción del mercado y la competencia, la descentralización de funciones y la delegación de poder a los agentes autónomos y la consideración del ciudadano como usuario-cliente, fueran la solución a sus problemas.

Sin embargo, a pesar de las críticas y problemas comentados anteriormente el establecimiento de la NGP ha cambiado notablemente la forma de actuación de las distintas Administraciones Públicas, volviéndolas más transparentes, más abiertas a los ciudadanos y con mayor flexibilidad en su gestión. 
BIBLIOGRAFÍA

AUCOIN, P. (1997) "Reforma administrativa en la gestión pública: Paradigmas, principios, paradojas y péndulos". MAP (eds.) (1997) Lecturas de Gestión Pública. Madrid, pp. 491-515.

BANCO MUNDIAL (1997) "El Estado en un mundo en transformación", Informe sobre le desarrollo mundial, Washington, D.C.

BARZELAY, M. (1992) Breaking Through Bureaucracy: A New Vision of Managing in Government. Berkeley and Los Angeles: University of California Press.

Boston, J. (1991) "The Theoretical Underpinnings of Public Sector Restructuring in New Zealand". J. Boston, J. MARTIN, J. PALlot \& P. WALSH (eds.) (1991) Reshaping the State. Auckland: Oxford University Press, pp. 1-26.

BoYNE, G.A. (1996) "Scale, Performance and the New Public Management: An Empirical Analysis of Local Authority Services", Journal of Management Studies, Vol. 33, № 6, pp. 809-826.

CUNILL, N. (1997) Repensando lo público a través de la sociedad. Nuevas formas de gestión pública y representación social. Caracas: CLAD, Nueva Sociedad.

Dawson, S. \& C. Dargie (2002) "New Public Management. A Discussion with Special Reference to UK Health". K. MCLAUGHLIN, S.P. OsBORNE \& E. FERLIE (eds.) (2002) The New Public Management: Current Trends and Future Prospects. London: Routledge, pp. 34-56.

Dimaggio, P. \& W. Powell (1991) "The Iron Cage Revisited: Institutional Isomorphism and Collective Rationality in Organizational Fields". P. DIMAGGIO y W. POWELL (eds.) (1991) The New Institutionalism in Organizational Analysis. Chicago University Press, pp. 63-81.

Dollery, B. \& Ch. Won LeE (2003) "New Public Management and Convergence in Public Administration Systems: A Comparison between Australia and the Republic of Korea", Working Paper Series in Economics. En línea: http://www.une.edu.au/febl/Economics/Publica tions/wps.htm (visita el 16/7/2003).

DunleaVy, P. \& C.C. Hood (1994) "From Old Public Administration to New Public Management", Public Money and Management, № 14:2, pp. 9-16.

EwALT, J.A.G. (2001) "Theories of Governance and New Public Management; Links to Understanding Welfare Policy Implementation", The Annual Conference of the American Society for Public Administration, Newark, NJ. 
FalCONER, P.K. (1999) Public Administration and the New Public Management: Lessons from the UK Experience. En línea: http://www. vus.uni-lj.si/Anglescina/FALPOR97.doc (visita el 29/7/2003).

FLYNN, N. (2002) "Explaining the New Public Management. The Important of Context". K. McLaughlin, S.P. Osborne \& E. FerLIE (Eds.) (2002) New Public Management. Current Trends and Future Prospects. London and New York: Routledge.

FUERTES FUERTES, I. (2004) La comparabilidad de la información contable pública en la Unión Europea. Valencia: Sindicatura de Comptes.

GIMENO RUIZ, A. (1997) "La descentralización de la gestión pública. Implicaciones contables", Actualidad Financiera, Número monográfico, $2^{\circ}$ trimestre, pp. 45-56.

GRUENING, G. (2001) "Origin and Theoretical Basis of New Public Management", International Public Management Journal, Vol. 4, Issue 1, pp. 1-25.

HOLMES, M. \& D. SHAND (1995) "Management Reform: Some Practitioner Perspectives on the Past Ten Years", Governance, Vol. 8, Issue 5, pp. 551-578.

Hood, C. (1991) "A Public Management for All Seasons?", Public Administration, $\mathrm{N}^{\circ} 69$, spring, pp. 3-19.

- (1995) "The New Public Management in the 1980s: Variation on a Theme", Accounting Organizations and Society, Vol. 20, $\mathrm{N}^{\circ} 2 / 3$, pp. 93-109.

- (2000) The Art of the State. Oxford: Oxford University Press.

- \& M. JACKSON (1991) Administrative Argument. Dartmouth: Aldershot.

HufTY, M. (1998) "Aux racines de la pensée comptable". M. HufTY (Dir.) (1998) La pensée comptable. État, Néolibéralisme, Nouvelle Gestion Publique. París: Presses Universitaires de France, pp.57-76.

Hughes, O.E. (1997) "La Nueva Gestión Pública". MINISTERIO DE LAS ADMINISTRACIONES PÚBLICAS - MAP (Ed.) (1997) Lecturas de Gestión Pública. Madrid: MAP.

- (2003) Public Management and Administration. An Introduction. New York: Macmillan Press Ltd.

KETTL, D.F. (1997) "The Global Revolution in Public Management: Living Themes, Missing Links", Journal of Policy Analysis and Management, $\mathrm{N}^{\circ}$ 16, pp. 446-462. 
LAN, Z. \& D.H. ROSENBLOOM (1992) "Public Administration in Transition?", Public Administration Review, November/December, Vol. 52, № 6, pp. 535-537.

LIKIERMAN, A. (1994) "Management Accounting in U.K. Central Government", Financial Accountability and Management, Vol. 10, $\mathrm{N}^{\circ} 2$, May, pp. 93115.

LÖFfLER, E. (1999) "The Value Public Management Evaluation from an International Perspective: Best Practice Cases Reconsidered", International Public Management Network Conference (IPMN), Siena, Italy.

LóPEZ CASASNOVAS, G. (1995) "Instrumentos al servicio de la gestión pública", V Congreso Nacional de Economía, Las Palmas de Gran Canaria, pp. 59-76.

LYNN, L.E.Jr. (1998) "The New Public Management: How to Transform a Theme into a Legacy", Public Administration Review, Vol. 58, № 3, pp. 231-237.

Mathiasen, D.G. (1999) "The New Public Management and its Critics", International Public Management Journal, Vol. 2, № 1, pp. 90-111.

MERRIEN (1998) "Misère de la nouvelle gestion publique". M. HufTY (Dir.) (1998) La pensée comptable. État, Néolibéralisme, Nouvelle Gestion Publique. París: Presses Universitaires de France, pp. 141-152.

MINISTERIO DE ADMINISTRACIONES PÚBLICAS - MAP (2000) Libro Blanco para la mejora de los Servicios Públicos: Una nueva Administración al servicio de los ciudadanos, $2^{a}$ ed.. Madrid: MAP.

MONTESINOS JULVE, V. (1996) "El control de la gestión pública por los órganos de control externo" II Seminario sobre Gestión Pública Local, Gijón, pp. 263-284.

- (1998) "Análisis contable de entidades públicas", IV Jornadas de Trabajo sobre Análisis Contable, ASEPUC, Palma de Mallorca, pp. 185-224.

- y A. Gimeno Ruiz (1998) "Contabilidad y Control en el marco de La Nueva Gestión Pública", IV Jornada de Trabajo sobre Contabilidad de Costes y Gestión, ASEPUC, Universitat Jaime I, Castelló de la Plana, pp. 145172.

MuÑoz Colomina, C.I. (1996) "Hacia una nueva gestión pública", Actualidad Financiera, Número monográfico, marzo, pp. 79-86.

ONRUBIA FERNÁNDEZ, J. (1995) "El modelo organizativo-institucional del sector público: la necesaria compatibilidad de los aspectos micro y macroeconómicos", ponencia presentada en el $V$ Congreso Nacional de 
Economía. Economía de los Servicios. Economía y Gestión de las Administraciones Públicas, Las Palmas de Gran Canaria, pp. 15-58.

ORGANIZACIÓN PARA LA COOPERACIÓN Y DESARROLLO ECONÓMICO - OCDE (1997) La transformación de la gestión pública. Las reformas en los países de la OCDE. Madrid: MAP.

- (1998) Public Management Reform and Economic and Social Development. Paris: PUMA.

OSBORNE, D. \& T. GAEBLER (1992) Reinventing Government: How the Entrepreneurial Spirit is Transforming the Public Sector. New York: Addison Wesley Publishing Company.

PABLOS RODRÍGUEZ, J.L. (1997) Gestión e información contable en las entidades locales. Madrid: Instituto de Contabilidad y Auditoría de Cuentas, Ministerio de Economía y Hacienda.

PolLITT, C. (1990) Managerialism and the Public Services. The AngloAmerican experience. Oxford: Blackwell.

- (1993) Managerialism and the Public Services: Cuts or Cultural Change in the 1990s, Second Edition. Oxford: Blackwell.

- \& G. BouCKAERT (2000) Public Management Reform: A Comparative Analysis. Oxford: Oxford University Press.

Poole, M.; R. MAnsfield, M. MARTInez-LuCio \& B. TuRner (1995) "Change and Continuities within The Public Sector: Contrasts between Public and Private Sector Managers in Britain and Effects of the «Thatcher Year»", Public Administration, Vol. 73, summer, pp. 271-286.

RAMIÓ MATAS, C. (2001) "Los problemas de la implantación de la nueva gestión pública en las Administraciones Públicas Latinas: Modelo de Estado y Cultura Institucional", Revista del CLAD: Reforma y Democracia, $N^{\circ} 21$, Caracas, Venezuela.

SAlVAdor SeRna, M. (2001) "El papel de las instituciones en la gestión de las administraciones públicas", Reforma y Democracia: Revista del CLAD, N²0, Caracas, pp. 73-108.

SÁNCHEZ MOTOS, E. (1997) "Administración Pública, ¿Reforma o Revolución?", Auditoría Pública, № 10, junio, pp. 42-45.

Suleiman, E. (2000) "Es Max Weber realmente irrelevante?", Gestión y Análisis de Políticas Públicas, № 17-18, INAP, Madrid.

ThOMSON, P. (1992) "Public Sector Management in a Period of Radical Change: 1979-1992", Public Money and Management, September, pp. $33-41$. 
Torres Pradas, L. y V. Pina Martínez (2003) "El NPM en la Europa Continental. ¿Convergencia o marketing público?", XII Congreso AECA, Cádiz.

VILLORIA, M. (1997) "Modernización administrativa y gobierno postburocrático". R. BAÑón y E. CARRILlo (comps.) (1997) La nueva Administración Pública. Madrid: Alianza Universidad Textos, pp. 77103.

VIVAS URIETA, C. (1998) "Indicadores de gestión para las entidades públicas", Boletín AECA, № 45, pp. 35-37.

WILLIAMSON, O. (1985) The Economic Institution of Capitalism: Firms, Markets, Relational Contracting. New York: Free Press.

WINBERG, A. (1994) "Reformas administrativas recientes y el papel cambiante de la evaluación de programas en Canadá", Jornadas "Tendencias y evolución de la Administración Pública en el mundo occidental: búsqueda de una mayor eficacia", Barcelona, Sindicaturas de Comptes de Catalunya, pp. 79-109. 